\title{
Sparse Statistical Deformation Model for the Analysis of Craniofacial Malformations in the Crouzon Mouse
}

Hildur Ólafsdóttir ${ }^{1,2}$, Michael Sass Hansen ${ }^{1}$, Karl Sjöstrand ${ }^{1}$, Tron A. Darvann ${ }^{2}$, Nuno V. Hermann ${ }^{2,3}$, Estanislao Oubel ${ }^{4}$, Bjarne K. Ersbøll ${ }^{1}$, Rasmus Larsen ${ }^{1}$, Alejandro F. Frangi ${ }^{4}$, Per Larsen ${ }^{2}$, Chad A. Perlyn ${ }^{5}$, Gillian M. Morriss-Kay ${ }^{6}$, and Sven Kreiborg ${ }^{2,3,7}$

${ }^{1}$ Informatics and Mathematical Modelling, Technical University of Denmark, Lyngby, Denmark

2 3D-Laboratory, School of Dentistry, University of Copenhagen; Copenhagen University Hospital; Informatics and Mathematical Modelling, Technical University of Denmark, Copenhagen, Denmark

${ }^{3}$ Department of Pediatric Dentistry and Clinical Genetics, School of Dentistry, Faculty of Health Sciences, University of Copenhagen, Copenhagen, Denmark

${ }^{4}$ Computational Imaging Lab, Department of Technology - D.326, Pompeu Fabra University, Barcelona, Spain

${ }^{5}$ Division of Plastic Surgery, Washington University School of Medicine, St. Louis, MO, USA

${ }^{6}$ Department of Physiology, Anatomy and Genetics, Oxford University, Oxford, UK

${ }^{7}$ Department of Clinical Genetics, The Juliane Marie Centre, Copenhagen University Hospital, Copenhagen, Denmark

\begin{abstract}
Crouzon syndrome is characterised by the premature fusion of cranial sutures. Recently the first genetic Crouzon mouse model was generated. In this study, Micro CT skull scannings of wild-type mice and Crouzon mice were investigated. Using nonrigid registration, a wild-type craniofacial mouse atlas was built. The atlas was registered to all mice providing parameters controlling the deformations for each subject. Our previous PCA-based statistical deformation model on these parameters revealed only one discriminating mode of variation. Aiming at distributing the discriminating variation over more modes we built a different model using Independent Component Analysis (ICA). Here, we focus on a third method, sparse PCA (SPCA), which aims at approximating the properties of a standard PCA while introducing sparse modes of variation. The results show that SPCA outperforms both ICA and PCA with respect to the Fisher discriminant, although many similarities are found with respect to ICA.
\end{abstract}

\section{Introduction}

Crouzon syndrome was first described nearly a century ago when calvarial deformities, facial anomalies, and abnormal protrusion of the eyeball were reported 
in a mother and her son [1. Later, the condition was characterised as a constellation of premature fusion of the cranial sutures (craniosynostosis), orbital deformity, maxillary hypoplasia, beaked nose, crowding of teeth, and high arched or cleft palate. Identification of heterozygous mutations in the gene encoding $f$ broblast growth factor receptor type 2 (FGFR2) have been found responsible for Crouzon syndrome [2. Recently a mouse model was created to study one of these mutations (FGFR2 Cys342Tyr) 3. Incorporating advanced small animal imaging techniques such as Micro $\mathrm{CT}$, allows for detailed examination of the craniofacial growth disturbances. Studying the craniofacial shape differences in detail contributes to the understanding of the syndrome, surgery planning and diagnosis in humans. A recent study, performing linear measurements on Micro CT scans, proved the mouse model applicable to reflect the craniofacial deviations occurring in humans with Crouzon syndrome 44. Previously, we have extended this study to assess the local deformations between the groups by constructing a deformable shape and intensity-based atlas of wild-type (normal) mouse skulls. Deforming this atlas to all mice, the craniofacial shape differences can be analyzed [5].

To analyse and interpret these deformations in a meaningful way, it is desirable to reduce the large number of dimensions and at the same time localise the growth deviations with respect to the atlas. This leads us to statistical deformation models (SDMs). These are closely related to statistical shape models but the fact that the whole correspondence field is modelled makes them more powerful. A standard PCA has been a popular approach to build SDMs (e.g. 6]7/8]) but recently different techniques have been applied, e.g. wavelet-based PCA [9].

With respect to the mouse study, PCA was previously performed 10. This analysis revealed only one discriminating mode of variation, mainly reflecting global differences between the groups. This kind of variation can be hard to interpret and in a recent study, we showed that applying Independent Component Analysis (ICA) to the deformation fields resulted in several discriminating modes, revealing the local differences between the groups. Sparse Principal Components Analysis (SPCA) [1] has proven successful when applied in shape modelling [12. In this paper we introduce the use of SPCA to build a Sparse Statistical Deformation Model and provide a comparison to a standard PCA and ICA with focus on the discriminative ability. We believe this is the first time SPCA is applied to statistically model deformation fields.

\section{Data Material}

Production of the $F$ f fr $2^{C 342 Y /+}$ and $F g f r 2^{C 342 Y / C 342 Y}$ mutant mouse (Crouzon mouse) has been previously described 3. All procedures were carried out in agreement with the United Kingdom Animals (Scientific Procedures) Act, guidelines of the Home Office, and regulations of the University of Oxford.

For three-dimensional (3D) CT scanning, 10 wild-type and $10 \mathrm{Fgfr} 2^{\mathrm{C} 342 \mathrm{Y} /+}$ specimens at six weeks of age (42 days) were sacrificed using Schedule I methods and fixed in $95 \%$ ethanol. They were sealed in conical tubes and shipped to the 


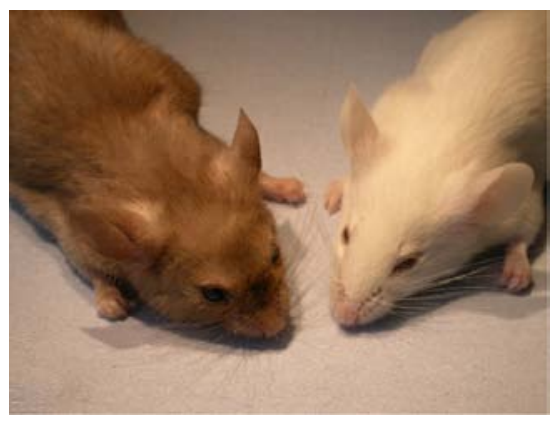

(a)

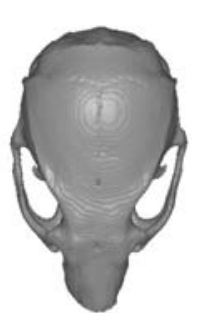

(b)

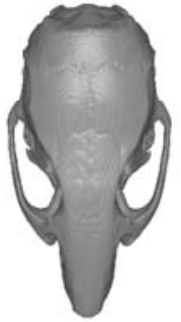

(c)

Fig. 1. (a) Photo of a Crouzon mouse (left) and a wild-type mouse (right). Skulls Extracted from CT images of (b) a Crouzon mouse, (c) wild-type mouse.

Micro CT imaging facility at the University of Utah. Images of the skull were obtained at approximately $46 \mu \mathrm{m} \times 46 \mu \mathrm{m} \times 46 \mu \mathrm{m}$ resolution using a General Electric Medical Systems EVS-RS9 Micro CT scanner. Fig. 1 shows an example of the living mice and the imaging data appearance.

\section{Methods}

The steps taken to automatically assess the local shape deviations between groups, statistically, from the Micro CT images are the following.

1. Build a craniofacial wild-type mouse atlas from the Micro CT's using nonrigid image registration

2. Match atlas to all 20 cases (wild-type and Crouzon mice) using nonrigid image registration

3. Use the resulting deformation parameters as input to a SPCA

\subsection{Atlas Building and Registration}

The first two steps of the procedure were presented in [5]. The nonrigid registration algorithm based on B-splines [1314] was applied. This algorithm uses a transformation model which is a combination of a global and a local transformation model, $\mathbf{T}(\mathbf{x})=\mathbf{T}_{\text {global }}(\mathbf{x})+\mathbf{T}_{\text {local }}(\mathbf{x})$. The global transformation model consists in our case of a rigid transformation matrix (with 6 degrees of freedom). The local transformation model describing the nonrigid part of the model is written by the tensor product of the 1D cubic B-splines,

$$
\mathbf{T}_{\text {local }}(x, y, z)=\sum_{l=0}^{3} \sum_{m=0}^{3} \sum_{n=0}^{3} B_{l}(u) B_{m}(v) B_{n}(w) \mathbf{c}_{i+l, j+m, k+n}
$$

where $\mathbf{c}$ are the parameters of the B-splines ordered in a $p_{x} \times p_{y} \times p_{y}$ lattice. $u, v$ and $w$ are the $(x, y, z)$ image coordinates translated into the lattice coordinates. 


\subsection{A Sparse Statistical Deformation Model}

The third step of the procedure listed above is the main focus of this paper. The control points (parameters) of the B-splines in Equation 1 provide a compact representation of the correspondence fields. As shown in [6] it is sufficient to perform a statistical analysis on these control points to obtain a compact description of the deformations. Using a common reference frame, e.g. an atlas, as the origin of the registrations, the control points for a subject reflect its local deviation from this reference frame. Concatenating the $3 \mathrm{D}$ control points for subject $i$ into a row vector $\mathbf{C}_{i}=\left[c_{1}, \ldots, c_{p}\right]$, where $p=3 p_{x} p_{y} p_{z}$, gives the $i$ th row of the $n \times p$ data matrix to analyse ( $n$ is the number of observations).

SPCA approximates the properties of a standard PCA while introducing sparsity in the modes of variation. Zou et al. 11] take advantage of formulating PCA as a regression problem leading to the $S P C A$ criterion

$$
\begin{gathered}
(\hat{\mathbf{A}}, \hat{\mathbf{B}})=\operatorname{argmin}_{\mathbf{A}, \mathbf{B}} \sum_{i=1}^{n}\left\|\mathbf{x}_{i}-\mathbf{A} \boldsymbol{B}^{T} \mathbf{x}_{i}\right\|^{2}+\lambda \sum_{j=1}^{k}\left\|\mathbf{b}_{j}\right\|^{2}+\sum_{j=1}^{k} \delta_{j}\left\|\mathbf{b}_{j}\right\|_{1} \\
\text { s.t. } \mathbf{A}^{T} \mathbf{A}=\mathbf{I}
\end{gathered}
$$

Here $\mathbf{x}_{i}$ denotes the $i$ th column of $\mathbf{X}^{T}$. This formulation assumes $k$ modes to be retained in the model. The columns of $\mathbf{B}$ represent the principal axes (loading vectors $\left.\mathbf{b}_{j}, j=1, \ldots, k\right)$ and $\mathbf{B}$ projects observation $i$ onto those axes. The matrix $\mathbf{A}$ takes the observation back to the original space. Hence, the first term measures the reconstruction error of the model. The second term, the L2 penalty is included to ensure a unique solution, also in cases where $p>n$, and the third term, L1 penalty, introduces sparsity. These two latter terms are adopted from Elastic Net regression [15]. The constraint weight, $\lambda$, must be chosen beforehand, and has the same value for all PCs, while $\delta$ may be set to different values for each PC, providing good flexibility.

The problem in Equation 2 is usually solved iteratively by fixing $\mathbf{A}$ in each iteration, solving for $\mathbf{B}$ using the LARS-EN algorithm [15] and recalculating $\mathbf{A}$. However, when we have $p \gg n$ as in our case, Zou et al. have shown that by letting $\lambda \rightarrow \infty, \mathbf{B}$ can be determined by soft thresholding 1

$$
\mathbf{b}_{j}=\left(\left|\mathbf{a}_{j}^{T} \mathbf{X}^{T} \mathbf{X}\right|-\frac{\delta_{j}}{2}\right)_{+} \cdot \operatorname{sign}\left(\mathbf{a}_{j}^{T} \mathbf{X}^{T} \mathbf{X}\right), \quad j=1,2, \ldots, k
$$

where $k$ is the number of modes and $\mathbf{a}_{j}$ is the $j$ th column of $\mathbf{A}$. This approach was taken here enforcing the same fixed level of sparsity in each loading vector by dynamically changing $\left(\delta_{j}\right)$ in each iteration. To maximise the total adjusted variance [11 explained by the SPCA, the modes were ordered allowing for perturbations as suggested in 12 .

Since the aim of our sparse deformation model is to discriminate between the two groups of mice the final ordering of modes was defined with respect to the Fisher discriminant. That is, the observations were projected onto the principal directions,

${ }^{1}(z)_{+}$denotes that if $z<0, z$ is set to 0 and if $z>=0, z$ is kept unchanged. The term is denoted hinge-loss. 
the Fisher discriminant between the groups calculated for each mode and the principal directions ordered with respect to decreasing Fisher discriminant score. In general, for class 1 and 2, the Fisher discriminant is defined as

$$
F=\frac{\left(\mu_{1}-\mu_{2}\right)^{2}}{\sigma_{1}^{2}+\sigma_{2}^{2}},
$$

where $\mu_{i}$ is the mean of class $i$ and $\sigma_{i}^{2}$ is the variance of class $i$.

\section{Experimental Results}

The accuracy of the image registration algorithm (registering the atlas to each of the 20 cases) is essential for the deformation model to be valid. In [5], the

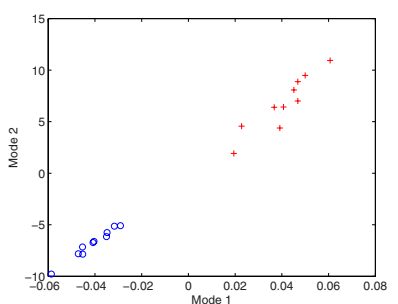

(a)

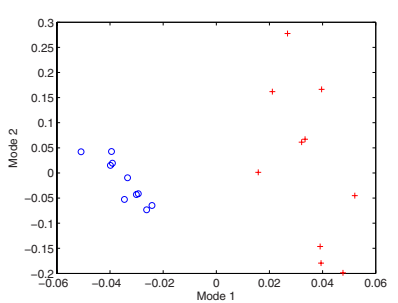

(d)

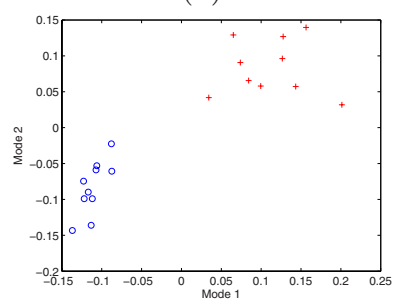

(g)

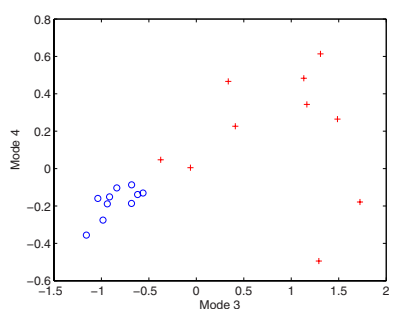

(b)

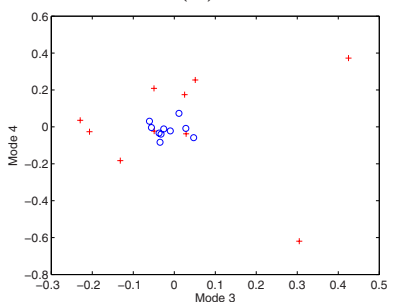

(e)

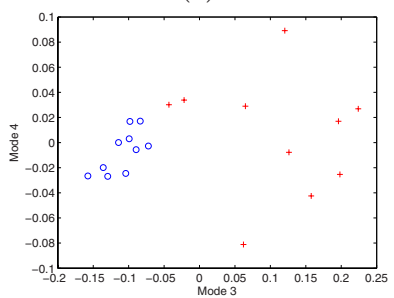

(h)

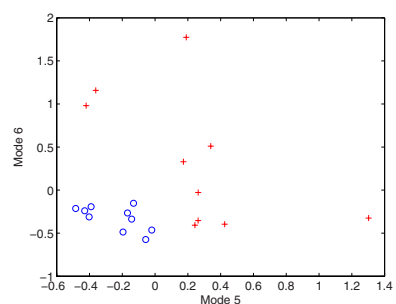

(c)

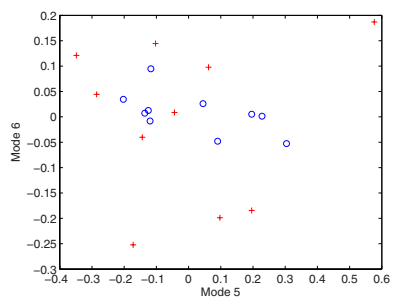

(f)

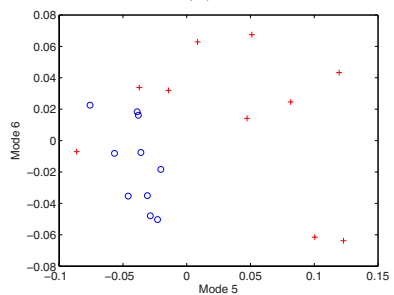

(i)

Fig. 2. Projection of observations into the space of the first six components (ordered by Fisher discriminant) using (a-c) SPCA, (d-f) PCA and (g-i) ICA. Crosses denote Crouzon cases while circles denote wild-type cases. (a,d,g) Mode 2 vs. mode 1; (b,e,h) Mode 4 vs. mode 3; (c,f,i) Mode 6 vs. mode 5. 


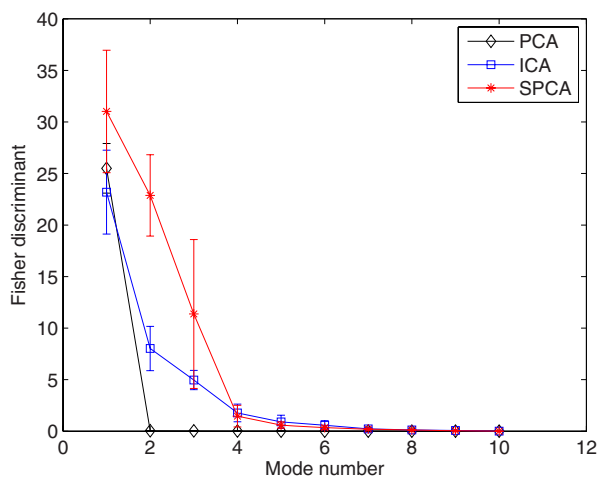

Fig. 3. The Fisher discriminant plotted vs. deformation mode number for PCA, ICA and SPCA. The values are obtained in a leave-one-out experiment providing the error bars (one standard deviation).

manual annotations from two observers were used to assess the registration accuracy. Using the optimal transformations from the image registrations, landmarks were obtained automatically. The landmark positions were statistically compared to those annotated by the human observers. This showed that the automatic method provided as good accuracy as the human observers and, moreover, it was more precise, judged from the significantly lower standard deviation.

The SPCA was applied to the matrix of control points $(p=21675)$. A threshold of 2000 points was used to obtain equal sparsity in each mode of variation. Fig. 2 (a-c) shows the observations projected onto the first six sparse principal directions (ordered by Fisher discriminant score). To evaluate the ability of the sparse SDM to assess the local group differences, it was compared to a standard PCA and our previous approach [16] using ICA [17. Fig. 2(d-i) shows scatter plots of the first six modes for ICA and PCA, sorted with respect to the Fisher discriminant.

The score plots already give an idea about the discrimination ability of the different approaches. To give a more quantitative measure, the Fisher discriminant was assessed in a leave-one-out fashion for all three approaches. This is plotted with error bars for each of the approaches in Fig. 3 .

With emphasis on the group differences, each mode of the sparse model was visualised by selecting the extremes from each group in model space (Fig. 2) and project back into the space of control points. This set of control points generated from the model was then applied to the atlas to obtain the deformed volumes of the two extremes. Subsequently the surfaces were extracted for visualisation. Fig. 4 shows mode 1,3,4 and 6. Mode 2 was excluded from this visualisation due to an overlap in variation with mode 1.

Deforming the atlas along the discriminating modes of the ICA model reveals many similarities between ICA and SPCA. To give an example Fig. 5 shows IC 5 which is closely related to SPC 4. 

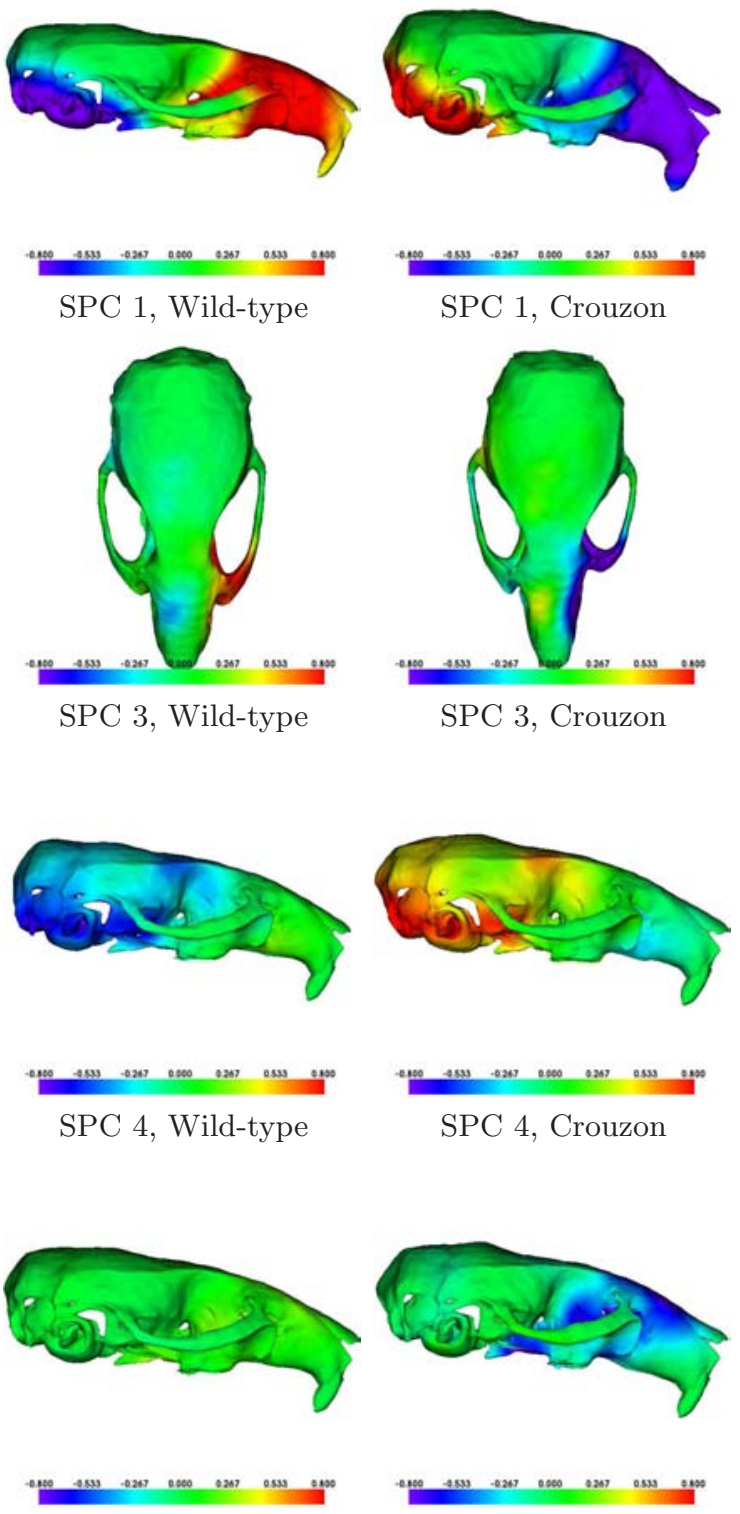

SPC 6, Wild-type

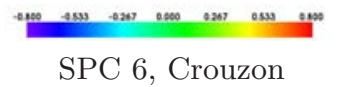

Fig. 4. Sparse Principal Deformation modes 1,3,4 and 6, visualised on surfaces after deforming atlas to the extremes of each mode. The colors are intended to enhance the regions where changes have occurred in the deformed surfaces. The colors denote displacement with respect to atlas (in $\mathrm{mm}$ ), with positive values (red) pointing outwards. 


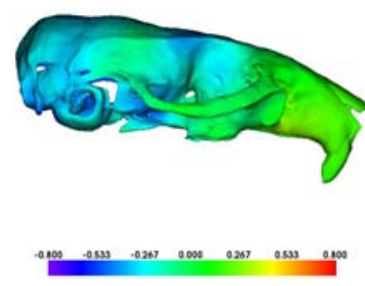

IC 5, Wild-type
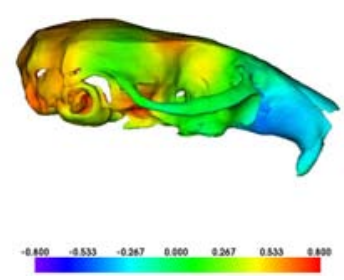

IC 5, Crouzon

Fig. 5. Independent Deformation mode 5 visualised on surfaces after deforming atlas to the extremes of the mode. The colors are intended to enhance the regions where changes have occurred in the deformed surfaces. The colors denote displacement with respect to atlas (in $\mathrm{mm}$ ), with positive values (red) pointing outwards.

\section{Discussion and Conclusions}

The score plots in Figure 2 indicate that both SPCA and ICA are capable of discriminating between the two groups in up to six deformation modes. The standard PCA only discriminates between the groups in the first mode. Figure 3 confirms these speculations. It is evident that PCA is only capable of discriminating between the groups in one mode of variation. SPCA performs slightly better than the ICA, but the ICA seems to be more robust judged from the error bars. Considering the low number of points in the sparse model, this is understandable.

Visualising the sparse deformation modes in Figure 4indicates that compared to wild-type mice, the skulls of Crouzon mice are higher and longer (SPC 1), are asymmetric with respect to zygoma and nose (SPC 3), have different shape of the middle ear and back of the head (SPC 4), and have an angulated cranial base (SPC 6). These observations correspond up to some degree with what has previously been seen in humans using manual measurements (see e.g. [18]). The asymmetric behaviour seen in SPC 3 can be explained by the full or partial fusion of cranial sutures at different sides and different times. The different shape of the middle ear and the increased angulation of the cranial base has not been reported in humans to our knowledge and may therefore be an important contribution to the understanding of the growth disturbances. The angulation was found in mice both using ICA [16] and PCA (with global transformation model extended to 9 DOFs) [10]. The difference in shape of the middle ear and back of the head was also captured by the ICA approach as seen in Figure 5. In fact SPC 4 and IC 5 are extremely similar, but SPCA seems to create slightly stronger evidence for the group difference. In general, the ICA modes introduce more noise than sparse PCA, since many elements are close to 0, while in SPCA, the sparsity property avoids this. Another advantage of SPCA is that it is solely based on second order statistics making it less committed than ICA, which uses higher order statistics.

In conclusion, with respect to discriminative ability, SPCA and ICA give similar results when applied to model deformations. Both of the approaches 
outperform a standard PCA. However, due to the simplicity and flexibility of SPCA, it should be the preferred method for this type of analysis.

\section{Acknowledgements}

For all image registrations, the Image Registration Toolkit was used under Licence from Ixico Ltd.

\section{References}

1. Crouzon, O.: Une nouvelle famille atteinte de dysostose cranio-faciale héréditère. Bull Mem. Soc. Méd Hôp Paris 39, 231-233 (1912)

2. Reardon, W., Winter, R.M., Rutland, P., Pulleyn, L.J., Jones, B.M., Malcolm, S.: Mutations in the fibroblast growth factor receptor 2 gene cause Crouzon syndrome. Nat. Genet. 8, 98-103 (1994)

3. Eswarakumar, V.P., Horowitz, M.C., Locklin, R., Morriss-Kay, G.M., Lonai, P.: A gain-of-function mutation of fgfr2c demonstrates the roles of this receptor variant in osteogenesis. Proc Natl Acad Sci, U.S.A. 101, 12555-12560 (2004)

4. Perlyn, C.A., DeLeon, V.B., Babbs, C., Govier, D., Burell, L., Darvann, T., Kreiborg, S., Morriss-Kay, G.: The craniofacial phenotype of the Crouzon mouse: Analysis of a model for syndromic craniosynostosis using 3D Micro CT. Cleft Palate Craniofacial Journal 43(6), 740-747 (2006)

5. Ólafsdóttir, H., Darvann, T.A., Hermann, N.V., Oubel, E., Ersbøll, B.K., Frangi, A.F., Larsen, P., Perlyn, C.A., Morriss-Kay, G.M., Kreiborg, S.: Computational mouse atlases and their application to automatic assessment of craniofacial dysmorphology caused by Crouzon syndrome. Journal of Anatomy (submitted) (2007)

6. Rueckert, D., Frangi, A.F., Schnabel, J.A.: Automatic construction of 3D statistical deformation models of the brain using nonrigid registration. IEEE Trans. on Medical Imaging 22(8), 1014-1025 (2003)

7. Mohamed, A., Zacharaki, E., Shen, D., Davatzikos, C.: Deformable registration of brain tumor images via a statistical model of tumor-induced deformation. Medical Image Analysis 10(5), 752-763 (2006)

8. Loeckx, D., Maes, F., Vandermeulen, D., Suetens, P.: Temporal subtraction of thorax CR images using a statistical deformation model. IEEE Transactions 22(11), 1490-1504 (2003)

9. Xue, Z., Shen, D., Davatzikos, C.: Statistical representation of high-dimensional deformation fields with application to statistically constrained 3D warping. Medical Image Analysis 10(5), 740-751 (2006)

10. Ólafsdóttir, H., Darvann, T.A., Ersbøll, B.K., Oubel, E., Hermann, N.V., Frangi, A.F., Larsen, P., Perlyn, C.A., Morriss-Kay, G.M., Kreiborg, S.: A craniofacial statistical deformation model of wild-type mice and Crouzon mice. In: International Symposium on Medical Imaging 2007, San Diego, CA, USA, The International Society for Optical Engineering (SPIE) (2007)

11. Zou, H., Hastie, T., Tibshirani, R.: Sparse principal component analysis. Technical report, Statistics Department, Stanford University (2004)

12. Sjöstrand, K., Stegmann, M., Larsen, R.: Sparse principal component analysis in medical shape modeling. In: International Symposium on Medical Imaging 2006, San Diego, CA, USA, vol. 6144. The International Society for Optical Engineering (SPIE) (2006) 
13. Rueckert, D., Sonoda, L.I., Hayes, C., Hill, D.L.G., Leach, M.O., Hawkes, D.J.: Nonrigid registration using free-form deformations: application to breast MR images. IEEE Trans. on Medical Imaging 18(8), 712-721 (1999)

14. Schnabel, J.A., Rueckert, D., Quist, M., Blackall, J.M., Castellano-Smith, A.D., Hartkens, T., Penney, G.P., Hall, W.A., Liu, H., Truwit, C.L., Gerritsen, F.A., Hill, D.L.G., Hawkes, D.J.: A generic framework for non-rigid registration based on non-uniform multi-level free-form deformations. In: Niessen, W.J., Viergever, M.A. (eds.) MICCAI 2001. LNCS, vol. 2208, pp. 573-581. Springer, Heidelberg (2001)

15. Zou, H., Hastie, T.: Regularization and variable selection via the elastic net. Journal of the Royal Statistical Society: Series B (Statistical Methodology) 67(2), 301-320 (2005)

16. Hansen, M.S., Olafsdóttir, H., Darvann, T.A., Hermann, N.V., Oubel, E., Larsen, R., Ersbøll, B.K., Frangi, A.F., Larsen, P., Perlyn, C.A., Morris-Kay, G.M., Kreiborg, S.: Estimation of independent non-linear deformation modes for analysis of craniofacial malformations in crouzon mice. In: Miles Wernick, J.A.F. (ed.) 2007 IEEE International Symposium on Biomedical Imaging, IEEE, Los Alamitos (2007)

17. Hyvärinen, A.: Survey on independent component analysis. Neural Computing Surveys 2, 94-128 (1999)

18. Kreiborg, S.: Crouzon Syndrome - A Clinical and Roentgencephalometric Study. Doctorate thesis, Institute of Orthodontics, The Royal Dental College, Copenhagen (1981) 